Published in Journal of Magnetic Resonance 207, 134-139 (2010) doi:10.1016/j.jmr.2010.08.018

\title{
Design and construction of an actively frequency- switchable RF coil for field-dependent Magnetisation Transfer Contrast MRI with fast field-cycling
}

Chang-Hoon Choi, James M.S. Hutchison, and David J. Lurie

Aberdeen Biomedical Imaging Centre, Biomedical Physics,

University of Aberdeen, Foresterhill, Aberdeen AB25 2ZD, UK

Correspondence to:

David J. Lurie, PhD

Aberdeen Biomedical Imaging Centre,

Biomedical Physics,

University of Aberdeen, Foresterhill, Aberdeen AB25 2ZD, UK

Tel: +44 (0) 1224 554061, Fax: +44 (0) 1224552514

E-mail: d.lurie@abdn.ac.uk 


\section{Abstract}

Magnetisation transfer contrast (MTC) is an important MR contrast-generating mechanism to characterise the MR-invisible macromolecular protons using an off-resonance pre-saturation RF irradiation pulse (or MT pulse). MTC MRI is normally implemented at a fixed magnetic field; however, it may be useful to evaluate changes of the MT effect as a function of external magnetic field strength $\left(B_{0}\right)$. In order to conduct field-dependent MTC experiments with a single MR system, two techniques are crucially needed. $B_{0}$ should be able to be switched between levels during irradiation of the MT pulse. At the same time, the resonance frequency of the RF coil $\left(f_{0}\right)$ should also be able to be shifted to the corresponding value. Switching $B_{0}$ is attained by the fast field-cycling technique, while in order to switch $f_{0}$, a specially designed multi-tunable RF coil is required. Here, we designed and constructed an actively frequency-switchable RF coil for frequencies at and below 2.5 MHz. The design employed PIN diodes, and enabled switching $f_{0}$ between five different values, with excellent impedance matching (approximately $-37 \mathrm{~dB} S_{11}$ reflection) and Q-factor of about 100 at each configuration.

Key words: Multi-tunable coil; PIN diode switch; Field cycling; Magnetization Transfer 


\section{Introduction}

Magnetisation Transfer Contrast (MTC) is a well-established MR contrast-generating mechanism, and is widely used for clarifying MR-invisible macromolecular information indirectly via MR-detectable free protons using their distinguishable NMR spectral linewidths [1,2]. In general, the restricted macromolecular protons are hardly detected by means of conventional MR techniques because of their very short $T_{2}$ relaxation times (few $\mu \mathrm{s}$ ) or very broad linewidths (few tens of $\mathrm{kHz}$ ). MTC MRI is typically implemented by applying an off-resonance radiofrequency (RF) irradiation pulse (or MT pulse) before acquiring MR data by ordinary MR pulse sequences, in order to irradiate the bound macromolecular protons whilst not affecting directly the free water protons. As a result of the MT pulse, magnetisation between both proton pools is exchanged and the signal intensity of mobile protons is decreased in relation to the amount of macromolecules.

The external magnetic field strength $\left(B_{0}\right)$ may play an essential role in developing magnetisation transfer contrast (MTC) imaging methods. MTC is normally employed at a fixed magnetic field, so many studies of MTC at medium and high magnetic field $(0.5 \mathrm{~T}$ and above) have been reported. There is, however, little information in the literature concerning the variation of the MT effect as a function of magnetic field, due to hardware limitations [24]. In order to explore field-dependent MTC experiments using a single MR instrument, two essential techniques are required, which enable simultaneously shifting both external magnetic field $\left(B_{0}\right)$ and the resonance frequency of an RF coil $\left(f_{0}\right)$ during MT pulse irradiation and returning them to the original condition during MR data acquisition, as shown in Fig. 1.

Switching of $B_{0}$ is achieved by fast field-cycling (FFC). FFC is a novel technique allowing $B_{0}$ to shift between levels rapidly during the pulse sequence. This makes it possible to perform a number of beneficial field-dependent studies and/or to provide new MR contrast mechanisms [5-7]. We have recently demonstrated the feasibility of combining FFC with MTC MRI using a new off-resonance irradiation method [8,9]. This technique should also be capable of conducting field-dependent MTC studies.

Switching of $f_{0}$ requires a multi-tunable RF coil. Previously, this type of coil has been built with various approaches. The first strategy to achieve multiple-resonant frequencies was to use dual or triple RF coils. Lurie et al. combined a birdcage coil (tuned for ${ }^{1} \mathrm{H}$ frequency) with an orthogonally positioned Alderman-Grant resonator (tuned for electron frequency) to detect free radical distribution via ${ }^{1} \mathrm{H}$ NMR signal using the Overhauser effect at low field [5]. 
Augath et al. built a triple-resonant RF coil for ${ }^{39} \mathrm{~K},{ }^{23} \mathrm{Na}$, and ${ }^{1} \mathrm{H}$ MR imaging of a rat head at 9.4 T. A saddle coil, a Helmholtz coil, and a printed flat mesh structure coil were respectively used to attain each configuration [10]. Their switchable frequencies were, however, limited to two (or three) values and coupling between the coils was also problematic. Another approach was to adapt a parallel LC trap circuit into a single RF coil. Schnall et al. included the trap circuit in series with the tuning capacitor into a surface coil which provided dual resonance frequencies for ${ }^{31} \mathrm{P}$ and ${ }^{1} \mathrm{H}$ NMR experiments at 3.0 T. They suggested that this method could be extended to more than two frequencies [11]. Similarly, Isaac et al. designed a low-pass birdcage coil by inserting the trap circuit into its legs in series in order to obtain both ${ }^{31} \mathrm{P}$ and ${ }^{1} \mathrm{H}$ resonance frequencies [12]. Shen et al. also demonstrated a double-tuned birdcage coil based on the method of a parallel trap circuit in order to present ${ }^{23} \mathrm{Na}$ and ${ }^{1} \mathrm{H}$ imaging at $1.5 \mathrm{~T}$ [13]. This method using the trap circuit could be used to avoid the coupling effect but the range of the switchable frequency was still limited. A third method introduced by Hwang and Hoult was to adjust tuning and matching capacitors by means of electric stepper motors [14]. Alternatively, other researchers have used varactor diodes. The capacitance of the varactor diode increases by increasing an applied reverse-bias voltage. Tugan Muftuler et al. used varactor diodes to build a low-pass birdcage resonator and achieved an automatically double-tunable resonator for ${ }^{1} \mathrm{H}$ and ${ }^{19} \mathrm{~F}$ imaging at $3.0 \mathrm{~T}$ [15]. de Alejo et al. also used varactor diodes in a saddle coil and attained an actively frequency controllable RF coil for ${ }^{1} \mathrm{H},{ }^{3} \mathrm{He}$, and ${ }^{19} \mathrm{~F}$ at $4.7 \mathrm{~T}$ [16]. They also addressed the problem that the Q-factor decreased with extending available frequencies. Hirata et al. constructed an electronically tunable surface coil using varactor diodes for tuning and matching systems in order to avoid frequency shifting and mismatching problems arising in in-vivo electron spin resonance experiments [17]. It may be possible to control tuning and matching for various nuclei of interest at high field; however, the frequency-switchable range of the varactor diodes may still be restricted to detect the nuclei at low field due to requiring higher capacitance.

In this work, we present the design and construction of an actively frequencyswitchable RF coil employing PIN diodes. PIN diodes are usually used not for switching two (or more) different frequencies but for tuning/detuning RF coils (a PIN diode becomes a conductor when forward biased whereas it becomes a high resistance when reverse bias is applied) $[18,19]$. Our design has enabled switching the coil's resonance frequency between five levels without limitation of the switchable frequency range [20]. 


\section{Methods}

A single-solenoid transmit/receive RF coil was designed and constructed for switchablefrequency operation around 2.5 $\mathrm{MHz}$. This type of coil provided sufficient $B_{1}$ field strength and Q-factor for MTC experiments, and horizontal sample access for use in our vertical- $B_{0}$ FFC-MRI scanner [5]. The coil was $150 \mathrm{~mm}$ in diameter and $75 \mathrm{~mm}$ in length, wound with 16 turns of $1.5 \mathrm{~mm}$ thick copper wire to avoid the skin depth effect, particularly dominant at low field. The winding density was increased at each end in order to improve $B_{1}$ field homogeneity. The coil was shielded by a cylinder of $50 \mu \mathrm{m}$ copper foil with $300 \mathrm{~mm}$ diameter and length. Figs. $2 \mathrm{a}$ and $2 \mathrm{~b}$ show photographs of the RF coil with and without shield.

A capacitive balanced matching network (Fig. 3) was used in order to equilibrate the coil's potential with respect to the ground leading to reduction of dielectric losses arising from the capacitive coupling with the sample (smaller frequency shift), and increase of Qfactor. In Fig. 3, $\mathrm{C}_{\text {match }}, \mathrm{C}_{\text {tune }}$, and $\mathrm{C}_{\text {sym }}$ are matching, tuning, and symmetric capacitors, respectively. The sum of the values of $\mathrm{C}_{\text {match }}$ and $\mathrm{C}_{\text {tune }}$ needs to be approximately equal to the capacitance $\left(\mathrm{C}_{\text {sym }}\right)$ in order to balance the circuit with good matching. The values of these capacitors were first calculated based on the coil's inductance $(42 \mu \mathrm{H}$ measured by means of a Q-meter (HP 4342A)) and desired resonance frequency (for example, 2.5 MHz). Trimmer capacitors which cover the calculated ranges of requirement were then selected. Two types of trimmer (Voltronics, USA), with capacitance ranges of $1.5 \mathrm{pF}$ to $55 \mathrm{pF}$ and $2 \mathrm{pF}$ to $120 \mathrm{pF}$, were used.

In order to increase the number of available resonance frequencies of the coil $\left(f_{0}\right)$, each PIN diode circuit (PIN diode itself (Infineon technologies, BAR64-05W) and trimmer capacitor) was attached in parallel with each $\mathrm{C}_{\text {tune }}$ and $\mathrm{C}_{\mathrm{sym}}$ of the balanced $\mathrm{RF}$ coil circuit. Fig. 3 shows the circuit diagram of the RF coil. The attached trimmer capacitors increase the overall capacitance of $\mathrm{C}_{\text {tune }}$ and $\mathrm{C}_{\mathrm{sym}}$ and, in turn decrease the coil's resonance frequency. As the detection field of our system was fixed at $59 \mathrm{mT}, 2.5 \mathrm{MHz}$ (corresponding Larmor frequency of $59 \mathrm{mT}$ ) was selected as the first frequency of the coil. This first $f_{0}$ was obtained either without attaching any PIN diode circuits or equivalently by attaching but disabling the PIN diode circuits in Fig. $3 \mathrm{~b}$. The second $f_{0}$ was adjusted using a set of all the PIN diode circuits (red line) (Fig. 3c) and third (orange lines) (Fig. 3d), fourth (green line) (Fig. 3e), and fifth (blue line) (Fig. 3f). Only one set of PIN diode circuits was active at a given time, while the others were in the off-state. Therefore, five different resonance frequencies were attained. 
In order to operate the PIN diodes, PIN diode drivers providing both forward and reverse bias voltages were required. The designs for these were modified and extended from reference [18]. The PIN diode drivers were built and assembled into an aluminium diecast box (55/110/65 $\mathrm{mm}$ in width/height/length) as shown in Fig. 4. Fig. 5 shows the circuit diagram of two of the PIN diode drivers. These drivers were powered by $9 \mathrm{~V}$ batteries (one for providing stable $+5 \mathrm{~V}$ and the others for $-12 \mathrm{~V}$ ) through voltage regulators (LM7805CT and L7912ACV). All power sources $(+5 \mathrm{~V}$ and $-12 \mathrm{~V})$ and ground were shared via each conductive spacer. Additional PIN diode drivers can easily be expanded for future applications. Activating these drivers is achieved by either manual switches 1 to 4 (for testing) or via TTL pulses from the MR console. For example, if a manual switch (SW 1) or a TTL pulse (TTL $A)$ is turned on, NPN transistor $\left(Q_{1}, 2 N 3904\right)$ in Fig. 5 is activated which allows current to flow from $+5 \mathrm{~V}$ to ground and this, in turn, activates PNP transistors $\left(\mathrm{Q}_{2}\right.$ and $\mathrm{Q}_{3}, 2 \mathrm{~N} 3906$ ). Consequently, $+5 \mathrm{~V}$ is transferred into the PIN diode driver outputs (DC (a) and DC (a')) to enable the pre-determined PIN diode set. On the other hand, if both input signals are switched off, all transistors are inactivated leading to an output of $-12 \mathrm{~V}$ which disables the PIN diode set.

DC and RF were split by homemade RF chokes (few $\mathrm{mH}$ ) located between the PIN diode and PIN diode driver outputs but closer to the PIN diodes. The RF chokes were about 5 to $7 \mathrm{~mm}$ in diameter and $5 \mathrm{~mm}$ in length, wound with many turns of $5 \mu \mathrm{m}$ thick copper wire in a plastic holder. A Q-meter (HP4342A) was used to measure the inductance of the RF chokes. Even though the RF chokes did not completely block RF (since the maximum inductance of RF chokes we could build in-house was approximately $4 \mathrm{mH}$ and above this value the choke became a capacitor), the frequency shift (few hundreds of $\mathrm{Hz}$ ) and Q-factor drop (few tens of the value) due to the RF leakage level was acceptable.

Four TTL channels were available for output from our MR console; however, only two of these were available for control of the RF coil. In order to double the available TTL lines, a TTL pulse divider was also built and assembled into the diecast box. Fig. 6 shows a truth table and logic diagram of the divider. This divider consisted of four AND logic gates (DM74LS08) and two Inverting gates (DM74LS04). This figure shows that only one $f_{0}$ is selected at one MT irradiation period.

The FFC-MTC-MRI pulse sequence was written using a C-like programming language on our system's commercial console (SMIS Ltd., UK). 
The coil was initially evaluated on the bench using a network analyser (Agilent Technologies, E5061A). MR imaging experiments were then carried out using a home-built field-cycling MRI system that uses a permanent magnet for detection and a co-axial resistive magnet for field-offset [5]. The operating field strength of the permanent magnet is fixed at $58.7 \mathrm{mT}$ and signal detection is always carried out at this field strength. However, the effective $B_{0}$ during other parts of the pulse sequence can be set at any value from zero to approximately $100 \mathrm{mT}$ by control from the pulse program.

A multi-sample phantom was prepared for the imaging experiments which consisted of $1 \%, 2 \%, 4 \%$, and $8 \%(w / v)$ agarose gel (Sigma-Aldrich, UK) samples. In addition, a sample only containing aqueous solution of $\mathrm{MnCl}_{2}$ (without agarose) was used as a control.

\section{Results}

Fig. 7 shows screen-shots of network analyser $S_{11}$ reflection measurements obtained using the actively frequency-switchable RF coil in five pre-determined configurations: $2.5 \mathrm{MHz}$, 2.4 MHz, 2.3 MHz, 2.2 MHz, and 2.1 MHz. The traces have been imported as Excel format. The centre frequency and span of the network analyser screen were fixed to $2.25 \mathrm{MHz}$ and $0.8 \mathrm{MHz}$, respectively. The pre-determined resonance frequency values of this resonator could initially be adjusted between $2.5 \mathrm{MHz}$ and $2.0 \mathrm{MHz}$ using the trimmer capacitors. These results in Fig. 7 indicate excellent $50 \Omega$ impedance matching (approximately $-37 \mathrm{~dB}$ return loss) and an unloaded Q-factor of about 100 at each frequency.

Fig. 8 shows the phantom images acquired with an interleaved (with and without MT irradiation) gradient-echo imaging pulse sequence. MT irradiation and signal detection were both performed at $2.5 \mathrm{MHz}$. The imaging parameters were: $1.5 \mathrm{~s}$ repetition time, $20 \mathrm{~ms}$ echo time, $180 \times 180 \mathrm{~mm}$ field of view, $128 \times 128$ pixel image matrix size, $20 \mathrm{~mm}$ slice thickness, $\mathrm{NEX}=8$ and the MT pulse parameter conditions were: $7.5 \mu \mathrm{T} B_{1}, 1.5 \mathrm{kHz}$ offset, and $270 \mathrm{~ms}$ MT pulse duration. In order to attain the $7.5 \mu \mathrm{T} B_{1}$ level, we have used a commercially available RF amplifier (Marconi Ltd., UK). The typical instantaneous transmit power level was approximately $18 \mathrm{~W}$ at the $7.5 \mu \mathrm{T} B_{1}$ level, with a duty cycle of $10 \%$, giving an average power in the MT irradiation of $1.8 \mathrm{~W}$. This is shared among 8 PIN diodes, giving an average power level of $0.225 \mathrm{~W}$ each, which is within the power-handling specification of the components used $(0.25 \mathrm{~W})$. 
In order to analyse the MT effects, regions of interest within the images were manually selected and magnetisation transfer ratios of each sample were calculated using the standard equation MTR $=1-\mathrm{M}_{\mathrm{s}} / \mathrm{M}_{0}$, where $\mathrm{M}_{\mathrm{s}}$ and $\mathrm{M}_{0}$ denote the signal intensities of the free protons measured with and without applying the MT saturation pulse, respectively. The MTR images were also acquired by pixel-by-pixel based image processing using MATLAB (MathWorks, Natick, MA, USA). Fig 8a shows the images without and with irradiating the MT pulse, and the MTR image (from left to right). Fig. $8 \mathrm{~b}$ presents the quantified MTR values calculated using the selected regions of interest from the images of the individual samples. Due to the absence of macromolecules in the control sample, we expected that the MT effect would be almost zero, but the frequency of the MT pulse was very close to the resonance frequency, so a small direct effect was present. Otherwise, the MT effects of the other samples increased with increasing concentration of agarose.

\section{Discussion}

We have demonstrated the design and construction of an automatically frequency-switchable RF coil. PIN diodes were used as electronic switches, which were attached in parallel into an initially balanced circuit with trimmer capacitors - one in $C_{\text {tune }}$ and the other in $C_{\text {sym. This }}$ enabled switching of the resonance frequency of the coil between five values with excellent impedance matching and reasonably high Q-factor. Due to the four inserted PIN diode circuits, the noise level of the coil was approximately doubled compared to that obtained without the PIN diode circuitry. This increase was mainly incurred by the imperfection of blocking the RF leakage via the RF chokes. In this work, we also have shown that the switchable frequency range was within a few hundred $\mathrm{kHz}$ at ultra-low field. However, the range can easily be extended by adding fixed capacitors in parallel with the trimmers and this has been tested. In addition, more available frequencies could be attained at the expense of decreasing the Q-factor, which is reduced due to the attachment of additional PIN diode circuits onto the RF coil.

In the future, we intend to conduct field-dependent MTC experiments using this coil with optimised MT pulse parameters and employing the FFC technique. In addition to its application for MTC, this design could also find use for multi-nuclear magnetic resonance as the switching time of this resonator is very short (few ns). 


\section{Acknowledgements}

The authors acknowledge financial support for the FFC-MRI project from Research Councils UK and the Engineering and Physical Sciences Research Council, under the Basic Technology scheme (grant number EP/E036775/1). We also thank Drs. Hugh Seton and Ioannis Lavdas for their helpful advice. 


\section{References}

[1] S.D. Wolff, R.S. Balaban, Magnetization transfer contrast (MTC) and tissue water proton relaxation in vivo, Magn. Reson. Med. 10 (1) (1989) 135-144.

[2] R.M. Henkelman, X. Huang, Q-S. Xiang, G.J. Stanisz, S.D. Swanson, M.J. Bronskill, Quantitative interpretation of magnetization transfer, Magn. Reson. Med. 29 (6) (1993) 759766.

[3] T.L. Ceckler, R.S. Balaban, Field dispersion in water-macromolecular proton magnetization transfer, J. Magn. Reson. Ser B. 105 (1994) 242-248.

[4] P. Martirosian, A. Boss, M. Deimling, B. Kiefer, C. Schraml, N.F. Schwenzer, C.D. Claussen, F. Schick, Systematic variation of off-resonance prepulses for clinical magnetization transfer contrast imaging at 0.2, 1.5, and 3.0 Tesla. Invest. Radiol. 43 (1) (2008) 16-26.

[5] D.J. Lurie, M.A. Foster, D. Yeung, J.M.S. Hutchison, Design, construction and use of a large-sample field-cycled PEDRI imager, Phys. Med. Biol. 43 (7) (1998) 1877-1886.

[6] S.E. Ungersma, N.I. Matter, J.W. Hardy, R.D. Venook, A. Macovski, S.M. Conolly, G.C. Scott, Magnetic resonance imaging with T1 dispersion contrast. Magn. Reson. Med. 55 (6) (2006) 1362-1371.

[7] J.K. Alford, B.K. Rutt, T.J. Scholl, W.B. Handler, B.A. Chronik, Delta relaxation enhanced mr: Improving activation - Specificity of molecular probes through R1 dispersion imaging. Magn. Reson. Med. 61 (4) (2009) 796-802.

[8] C.-H. Choi, G.R. Davies, D.J. Lurie, Off-resonance magnetisation transfer contrast MRI using fast field-cycling technique, In Proceedings of the $17^{\text {th }}$ Annual Meeting of ISMRM, Honolulu, USA (2009) p.2747.

[9] C.-H. Choi, G.R. Davies, D.J. Lurie, Off-resonance magnetisation transfer contrast (MTC) MRI using fast field-cycling (FFC), J. Magn. Reson. 204 (1) (2010) 145-149.

[10] M. Augath, P. Heiler, S. Kirsch, L.R. Schad, In vivo ${ }^{39} \mathrm{~K},{ }^{23} \mathrm{Na}$ and ${ }^{1} \mathrm{H}$ MR imaging using a triple resonant RF coil setup, J. Magn. Reson. 200 (2009) 134-136.

[11] M.D. Schnall, V. Harihara Subramanian, J.S. Leigh, B. Chance, A new double-tuned probe for concurrent ${ }^{1} \mathrm{H}$ and ${ }^{31} \mathrm{P}$ NMR, J. Magn. Reson. 65 (1985) 122-129. 
[12] G. Isaac, M.D. Schnall, R.E. Lenkinski, K. Vogele, A design for a double-tuned birdcage coil for use in an integrated MRI/MRS examination, 89 (1990) 41-50.

[13] G.X. Shen, F.E. Boada, K.R. Thulborn, Dual-frequency, dual-quadrature, birdcage RF coil design with identical B1 pattern for sodium and proton imaging of the human brain at 1.5 T, Magn. Reson. Med. 38 (1997) 717-725.

[14] F. Hwang, D.I Hoult, Automatic probe tuning and matching, Magn. Reson. Med. 39 (1998) 214-222.

[15] L. Tugan Muftuler, G. Gulsen, K.D. Sezen, O. Nalcioglu, Automatic tuned MRI RF coil for multinuclear imaging of small animals at 3T, J. Magn. Reson. 155 (2002) 39-44.

[16] R.P. de Alejo, C. Garrido, P. Villa, I. Rodriguez, J.J. Vaquero, J. Ruiz-Cabello, M. Cortijo, Automatic tuning and matching of a small multifrequency saddle coil at $4.7 \mathrm{~T}$, Magn. Reson. Med. 51 (2004) 869-873.

[17] H. Hirata, T. Walczak, H.M. Swartz, Electronically tunable surface-coil-type resonator for L-band EPR spectroscopy, J. Magn. Reson. 142 (2000) 159-167.

[18] J.R. Garbow, C. Mcintosh, M.S. Conradi, Actively decoupled transmit-receive coil-pair for mouse brain MRI, Concepts Magn. Reson. B, 33 (4) (2008) 252-259.

[19] E.A. Barberi, J.S. Gati, B.K. Rutt, R.S. Menon, A transmit-only/receive-only (TORO) RF system for high-field MRI/MRS applications, Magn. Reson. Med. 43 (2000) 284-289.

[20] C.-H. Choi, I. Lavdas, J.M.S. Hutchison, D.J. Lurie, Design and construction of an actively frequency-switchable RF coil for magnetisation transfer contrast MRI using fast field-cycling, In Proceedings of the $18^{\text {th }}$ Annual Meeting of ISMRM, Stockholm, Sweden (2010) p.3923. 

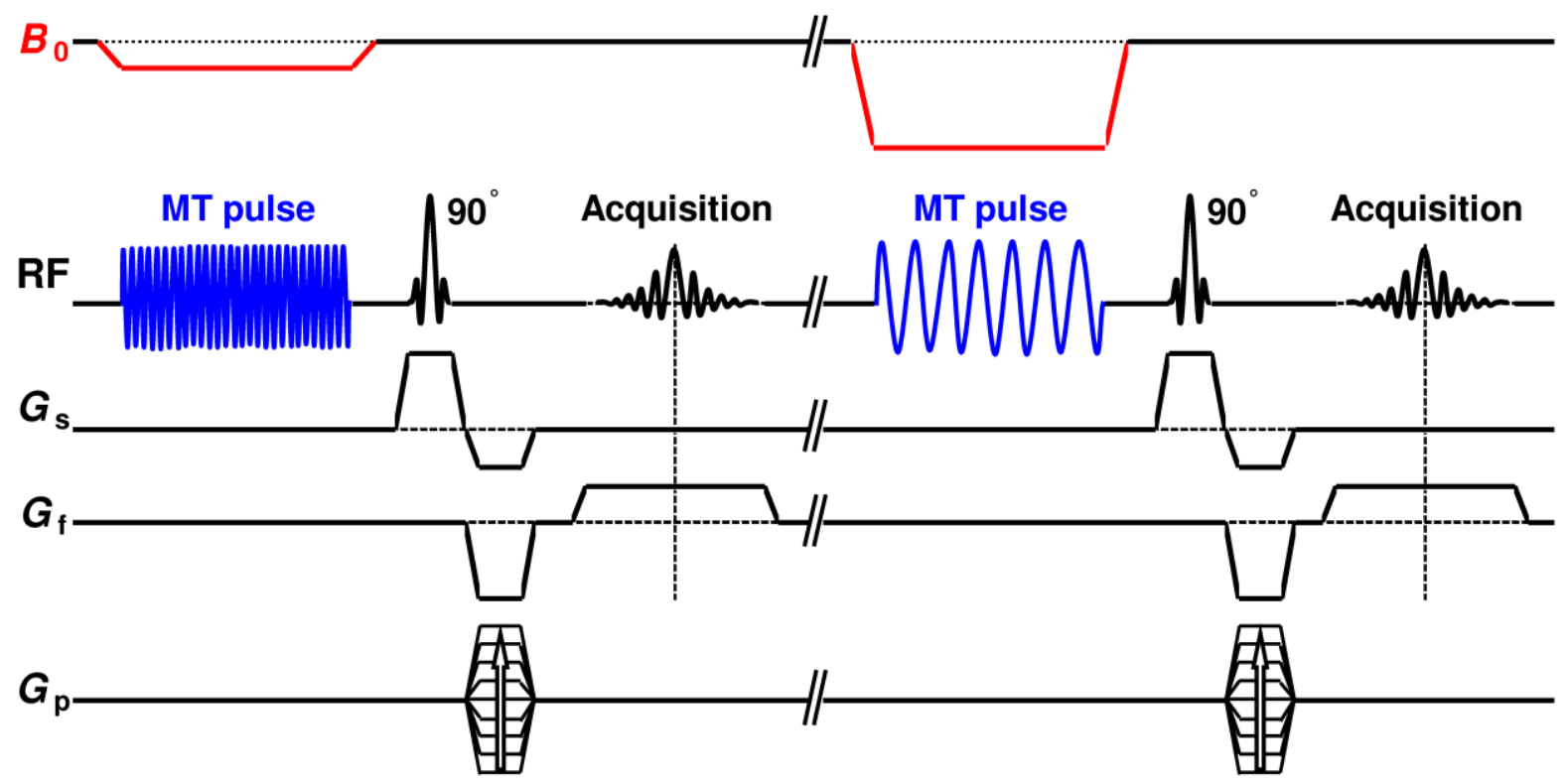

Fig. 1. Example of a pulse sequence for field-dependent MTC experiments showing that both main magnetic field strength $\left(B_{0}\right)$ and the resonance frequency of the RF coil $\left(f_{0}\right)$ are concurrently shifted to pre-determined levels during MT irradiation but returned to the original value during NMR acquisition.

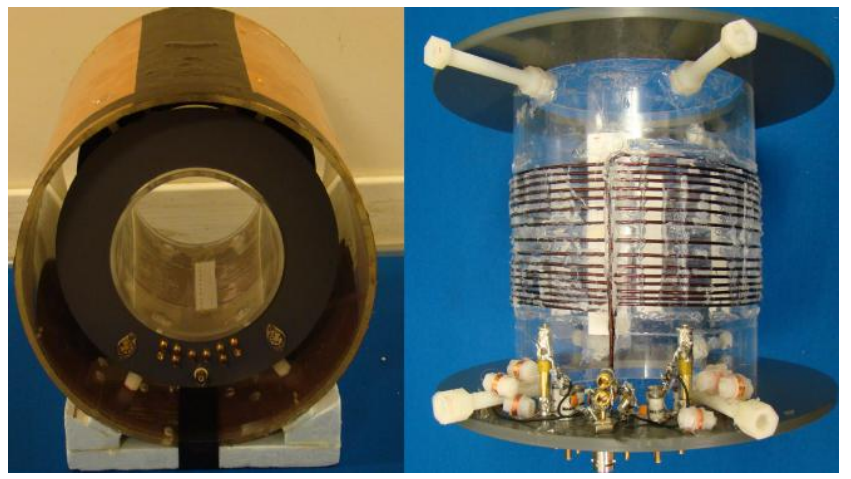

Fig. 2. Photographs of the RF resonator (a) with and (b) without shield. 

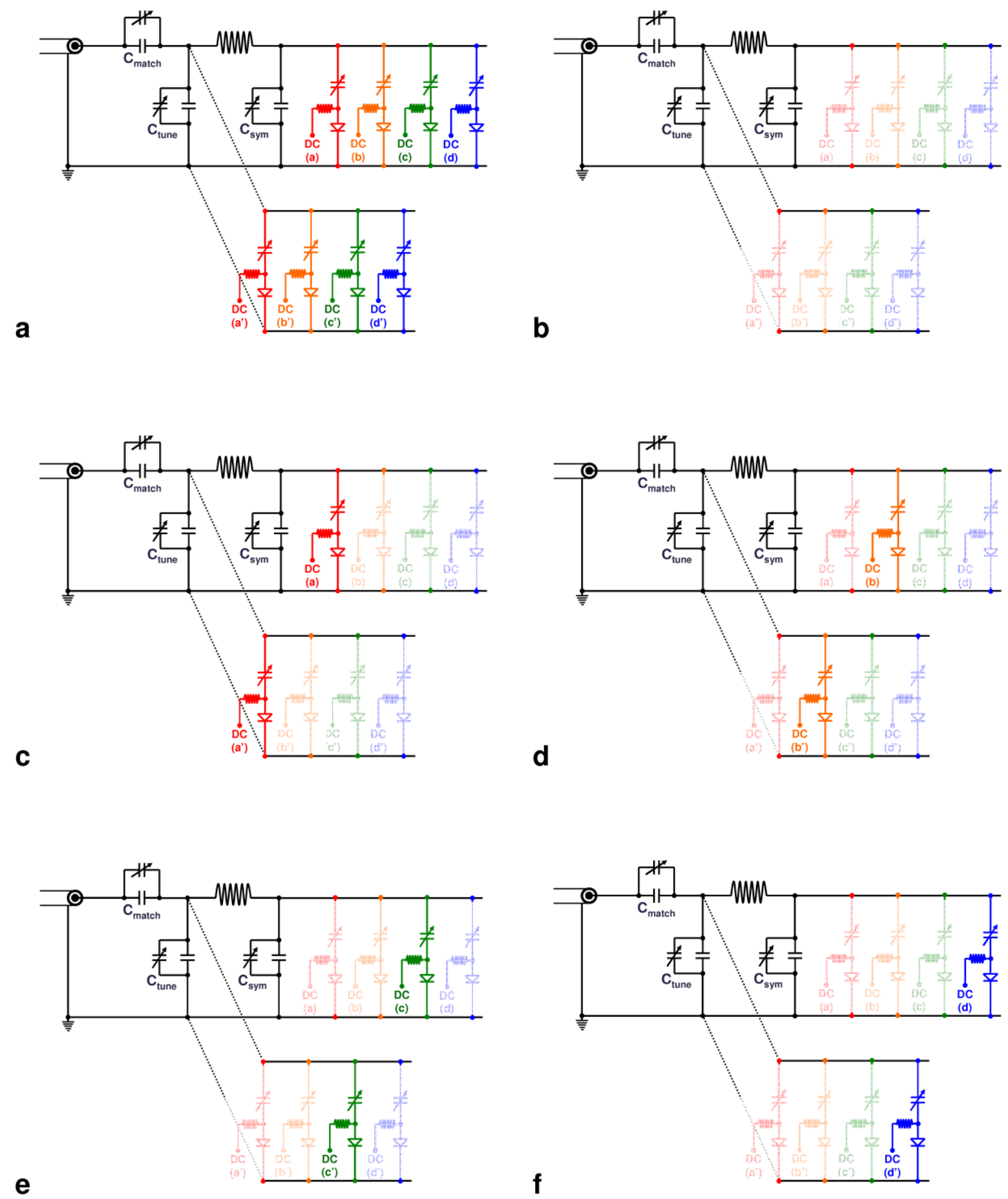

Fig. 3. (a) Overall schematic circuit diagram for the proposed RF coil. The attached PIN diode set is sequentially turned on while other PIN diodes are turned off. (b) The first frequency is achieved by providing $-12 \mathrm{~V}$ to all PIN diodes, (c) the second frequency is attained by applying $+5 \mathrm{~V}$ to the first PIN diode set (thereby, turning on the selected PIN diodes and adding the amount of capacitance of trimmer capacitors into total $\mathrm{C}_{\text {tune }}$ and $\mathrm{C}_{\text {sym }}$ ) but $-12 \mathrm{~V}$ to other PIN diode sets, and (d) third, (e) fourth, and (f) fifth. 


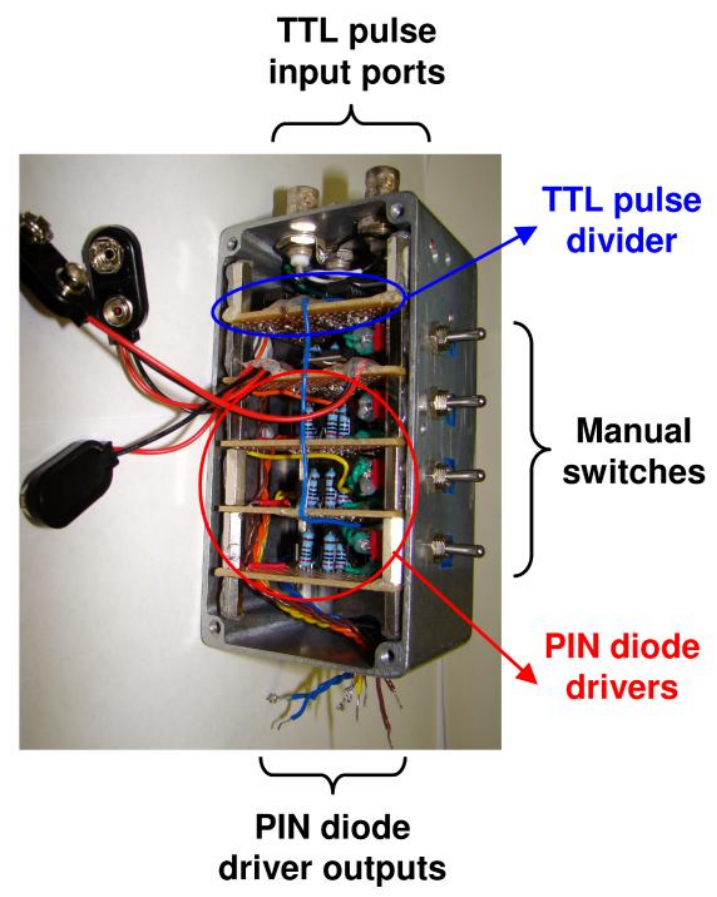

Fig. 4. Photograph of the diecast box containing four PIN diode drivers and one TTL pulse divider.

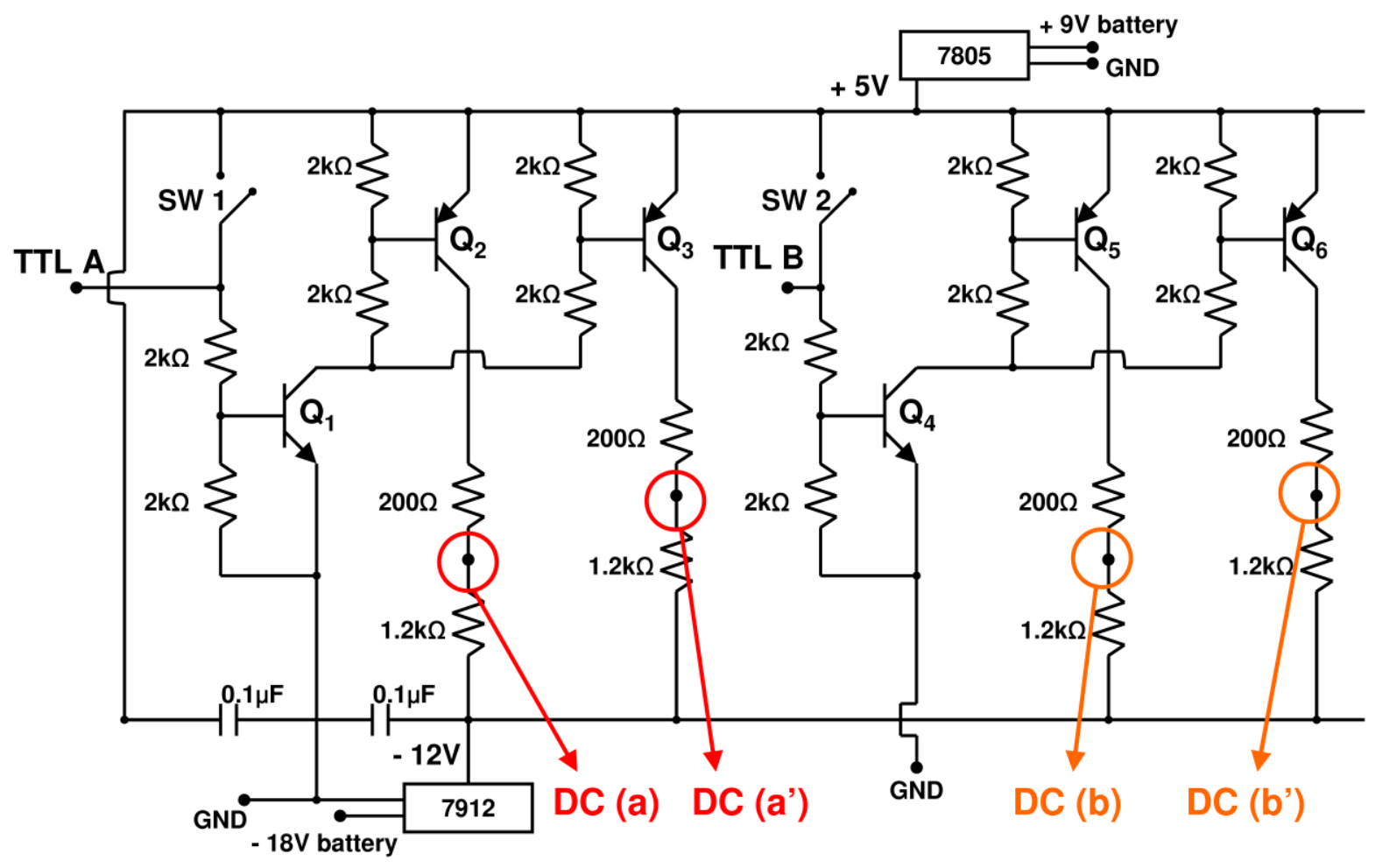

Fig. 5. $\mathrm{PIN}$ diode driver circuit diagram $\left(\mathrm{Q}_{1}\right.$ and $\mathrm{Q}_{4}-\mathrm{NPN}$ transistors and $\mathrm{Q}_{2}, \mathrm{Q}_{3}, \mathrm{Q}_{5}$, and $\mathrm{Q}_{6}$ - PNP transistors). 


\begin{tabular}{cccc|cccc|c} 
TTL1 & TTL 2 & TTL 3 & TTL 4 & TTL A & TTL B & TTL C & TTL D & Frequency \\
\hline $\mathrm{X}$ & 0 & $\mathrm{X}$ & 0 & 0 & 0 & 0 & 0 & Disable \\
$\mathrm{X}$ & 0 & $\mathrm{X}$ & 1 & 0 & 0 & 0 & 0 & $2.5 \mathrm{MHz}$ \\
0 & 1 & 0 & 1 & $\mathbf{1}$ & 0 & 0 & 0 & $2.4 \mathrm{MHz}$ \\
0 & 1 & 1 & 1 & 0 & 1 & 0 & 0 & $2.3 \mathrm{MHz}$ \\
1 & 1 & 0 & 1 & 0 & 0 & 1 & 0 & $2.2 \mathrm{MHz}$ \\
1 & 1 & 1 & 1 & 0 & 0 & 0 & 1 & $2.1 \mathrm{MHz}$
\end{tabular}

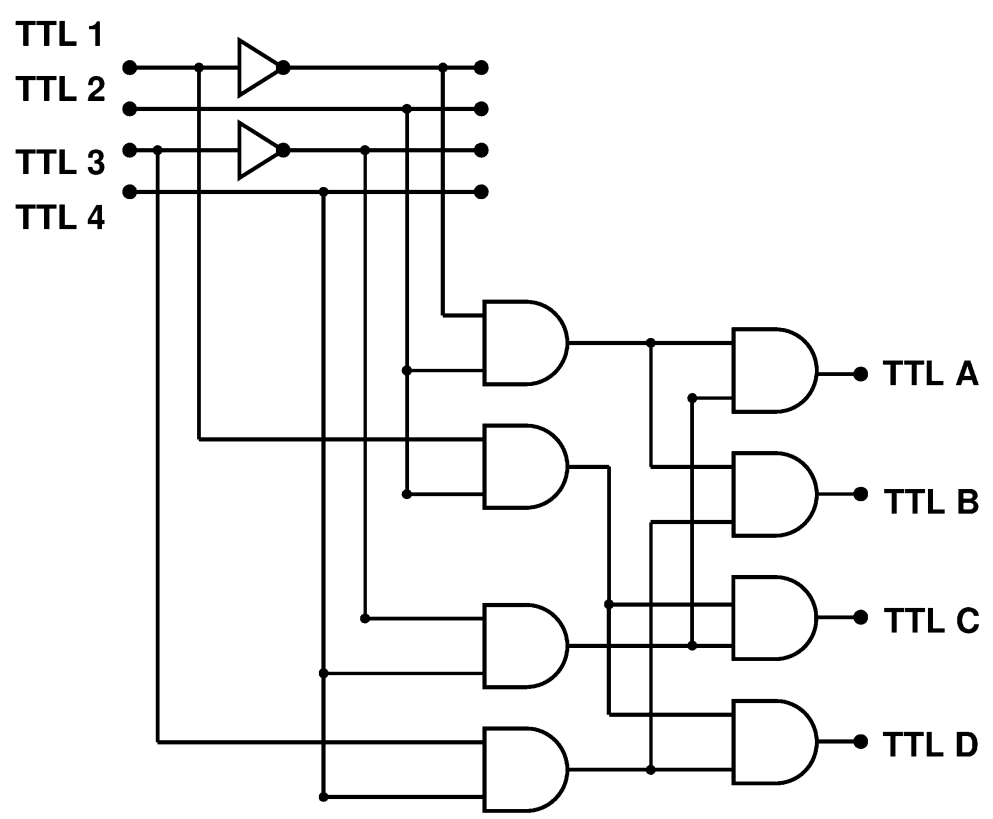

Fig. 6. Truth table and logic diagram of the TTL pulse divider. 


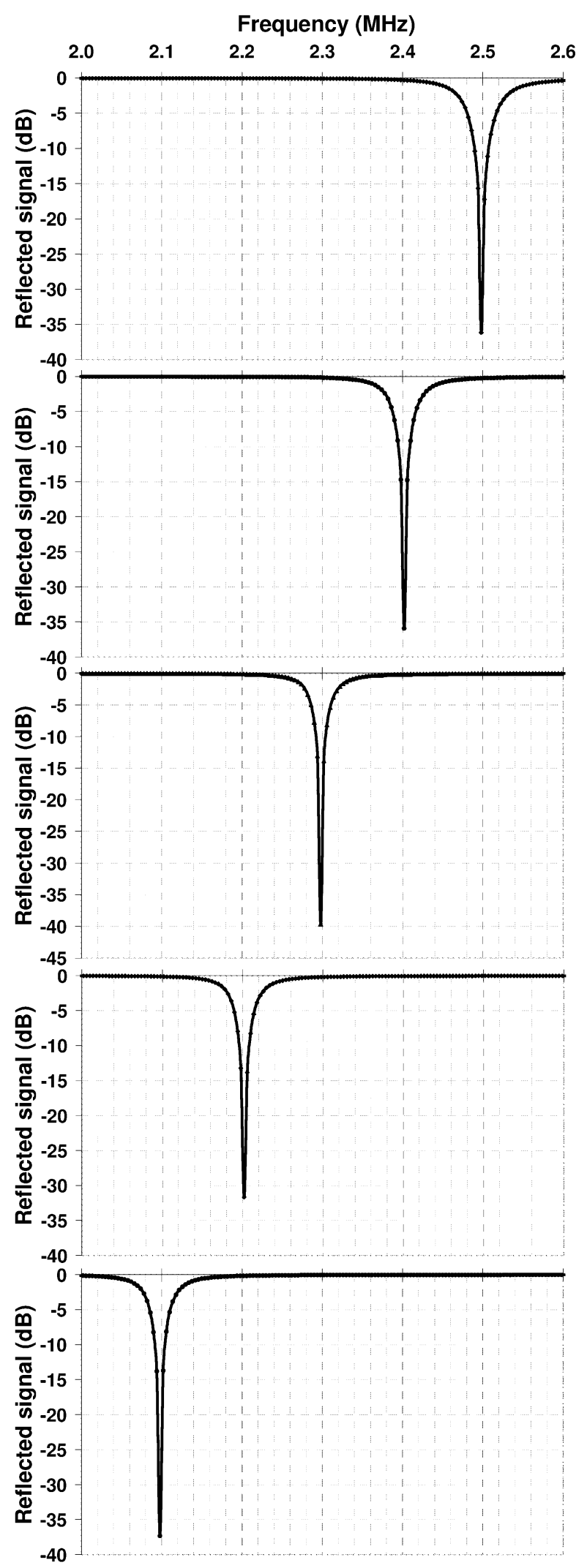

Fig. 7. Snap-shots of network analyser $S_{11}$ measurements obtained at the five preset configurations: 2.5 MHz, 2.4 MHz, 2.3 MHz, 2.2 MHz, and 2.1 MHz (From top to bottom). 

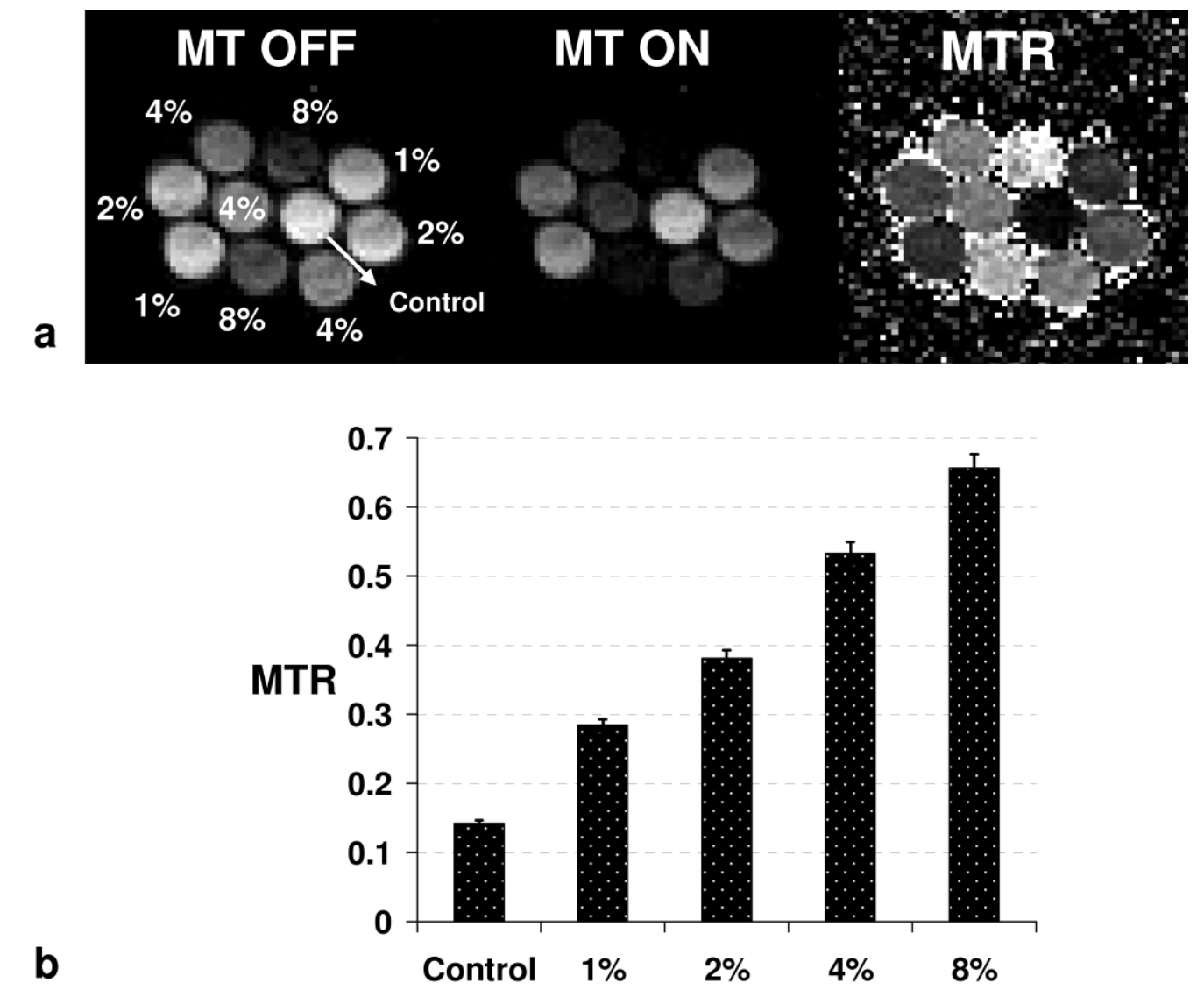

Fig. 8. (a) Phantom images obtained using the frequency-switchable coil at the operating frequency of $2.5 \mathrm{MHz}$ before (MT OFF) and after (MT ON) MT irradiation and the last MTR image is processed by MATLAB. (b) Quantified MTR values using the selected regions of interest from the images of the individual samples. 